\title{
Mycoplasma pneumoniae Associated Mucositis
}

\section{Salma Fahmidha ${ }^{1} \cdot$ Manobalan Karunanandhan $^{1}$ (D) $\cdot$ Lalitha Munigangaiah ${ }^{2} \cdot$ Rangan Srinivasaraghavan $^{2}$}

Received: 2 August 2019 / Accepted: 15 June 2020 / Published online: 26 June 2020

(C) Dr. K C Chaudhuri Foundation 2020

To the Editor: Mycoplasma pneumoniae associated mucositis (MPAM) is a rare extrapulmonary manifestation of M. pneumoniae infection. The disease is characterised by the presence of mucosal involvement of the oral cavity, eyes and genitalia, with or without skin involvement. Skin involvement is seen in 20-25\% of patients [1]. A 6-y-old boy presented with fever, cough, painful lip ulcers and eye discharge for $4 \mathrm{~d}$. On examination the child was well preserved. Cutaneous examination showed erythematous macules and few papules on the arm. There were superficial ulcers with hemorrhagic crusts on both the lips. Conjunctivae appeared congested and associated with mild purulent discharge. Genitalia and other systemic examinations including respiratory examination were normal. Lab investigations revealed neutrophilia, raised ESR and CRP positivity. IgM serology was positive for $M$. pneumoniae. Chest Xray showed right sided minimal infiltrates. A diagnosis of MPAM was made. The child was treated with syrup azithromycin $200 \mathrm{mg}$ once daily for $5 \mathrm{~d}$. Saline soaks were given on the lesions. Fever and cough subsided following treatment. Skin lesions healed in one week without any sequelae.

M. pneumoniae is a common cause of community acquired pneumonia in children. M. pneumoniae infection associated with ocular, oral mucositis and urogenital lesions with minimal or no skin involvement is rare. As it mimics erythema multiforme/Stevens Johnson syndrome (SJS), it was named as atypical/incomplete SJS in the past [2]. Unlike SJS/Toxic epidermal necrolysis wherein skin involvement is predominant, MPAM has nil or only 20-25\% skin involvement [1].

The exact mechanism by which $M$. pneumoniae causes cutaneous manifestation is not known. The proposed mechanisms include immune complex-mediated vascular injury,

Manobalan Karunanandhan

mano.jipmer@gmail.com

1 Department of Dermatology, Venereology and Leprosy, Mahatma Gandhi Medical College and Research Institute, Puducherry, India

2 Department of Pediatrics, Mahatma Gandhi Medical College and Research Institute, Puducherry, India cell-mediated immune response/cytotoxic injury to epithelial cells, and autoimmune mechanisms [3].

Canavan et al. proposed diagnostic criteria for MPAM which is as follows: i) Skin detachment $<10 \%$ BSA (Body surface area), ii) Lesions on two or more mucosal sites, iii) Few vesiculobullous/scattered atypical targets, iv) Presence/ absence of targetoid lesions, v) Evidence of $M$. pneumoniae infection (clinical features $+\operatorname{IgM}$ serology/PCR and/or serial cold agglutinins) $[4,5]$.

The treatment of MPAM includes macrolide, fluoroquinolone and tetracycline antibiotics with supportive care. Few studies have recommended IVIG and corticosteroids for nonresponsive patients [3]. MPAM has an excellent prognosis with a very low risk of recurrence $(<10 \%)$, complete recovery and a very rare need for intensive care treatment [3]. Rare sequelae that may occur are mucosal synechiae and pigmentary changes.

\section{Compliance with Ethical Standards}

Conflict of Interest None.

\section{References}

1. Schalock PC, Dinulos JGH. Mycoplasma pneumoniae-induced cutaneous disease. Int J Dermatol. 2009;48:673-80; quiz 680-1.

2. Schalock PC, Dinulos JGH. Mycoplasma pneumoniae-induced Stevens-Johnson syndrome without skin lesions: fact or fiction? J Am Acad Dermatol. 2005;52:312-5.

3. Bressan S, Mion T, Andreola B, Bisogno G, Da Dalt L. Severe mycoplasma pneumoniae-associated mucositis treated with immunoglobulins. Acta Paediatr. 2011;100:e238-40.

4. Ferwerda A, Moll HA, de Groot R. Respiratory tract infections by mycoplasma pneumoniae in children: a review of diagnostic and therapeutic measures. Eur J Pediatr. 2001;160:483-91.

5. Canavan TN, Mathes EF, Frieden I, Shinkai K. Mycoplasma pneumoniae-induced rash and mucositis as a syndrome distinct from Stevens-Johnson syndrome and erythema multiforme: a systematic review. J Am Acad Dermatol. 2015;72:239-45.

Publisher's Note Springer Nature remains neutral with regard to jurisdictional claims in published maps and institutional affiliations. 